\title{
Processing of clustered DNA damages by Nucleotide Excision Repair pathway
}

\author{
Natalia Lukianchikova \\ ICBFM SB RAS, Novosibirsk, Russia \\ lunata9@yandex.ru \\ Irina Petruseva
}

\author{
ICBFM SB RAS, Novosibirsk, Russia \\ irapetru@niboch.nsc.ru \\ Alexander Lomzov \\ ICBFM SB RAS, Novosibirsk, Russia \\ lomzov@niboch.nsc.ru
}

\author{
Olga Lavrik \\ ICBFM SB RAS, Novosibirsk, Russia \\ lavrik@niboch.nsc.ru
}

\begin{abstract}
Nucleotide excision repair (NER) is a multistep process that eliminates a wide range of damages in DNA, including UV photoproducts and base modifications by many carcinogenic and chemotherapeutic agents [1]. Clustered DNA damages are defined as two or more damages situated within one to two helical turns of dsDNA [2]. In this study, we examined how the presence of AP site analog within a cluster with bulky damage in DNA impacts on functioning of Nucleotide Excision Repair machinery of mammalian cell.
\end{abstract}

Keywords - DNA repair, Nucleotide Excision Repair.

Motivation and Aim

\section{Motivation}

We aim to analyze systematically the properties of the model DNAs bearing clustered damages. The main goal was to identify how the efficiency of recognition and removal of damages forming a cluster depends on both the structure and the distance between damages.

$$
\text { Aim }
$$

Earlier we have developed a mathematical model of cardiomyocyte electro-mechanical function [1] that predicted a significant role of the intra- and extracellular mechanical factors in arrhythmogenesys. Model prediction was verified in experiments on papillary muscles from the right ventricle of guinea pigs overloaded with calcium [2].

\section{Methods}

In order to evaluate the efficiency of NER we utilized the approach based on reproducing the repair reaction by mixing protein extracts from mammalian cells with modellinear DNAs, bearing clustered damages. We havesynthesized the set of long linear DNAs (137 bp), containing nFlu, the nonnucleoside bulky lesion, recognized and processed by NER system, and DEG, AP site analog). We also used the fluorescence anisotropy measurements for evaluation XPC affinity to the cluster-containing DNAs. Using photoaffinity labeling we have analyzed the interactions between XPD protein and the DNA probes bearing clustered structures. Molecular dynamics simulations have revealed structural for the differences observed.

\section{Results}

Results of fluorescence anisotropy measurements have shown that XPC has a significantly increased affinity to the cluster-containing DNAs. We have demonstrated that the NER-catalyzed excision of the DNA fragments that contain $\mathrm{nFlu}$ is fully abrogated in the presence of DEG insert in the certain positions of the complementary strand. The excision efficiency from the DNAs, containing the bulky lesion and the AP-site analog in one strand was not impeded. The experimental data together with Molecular Dynamic simulation results contribute to an understanding of the repair mechanisms of bulky adducts-containing clusters of different topologies in mammalian cell.

\section{ACKNOWLEDGMENT}

Supported by RFBR grant No. 19-04-00018.

\section{REFERENCES}

[1] Orlando D. Schärer O. (2013) Nucleotide Excision Repair in Eukaryotes. Cold Spring Harb Perspect Biol. 5(10): a012609.

[2] Sage E., Harrison L. (2011) Clustered DNA lesion repair in eukaryotes: relevance to mutagenesis and cell survival. Mutat Res. 711(1-2): 123133. 